\title{
Morphine Measurement
}

National Cancer Institute

\section{Source}

National Cancer Institute. Morphine Measurement. NCI Thesaurus. Code C74883.

The determination of the amount of morphine present in a sample. 